\title{
Co-Isolation of Cytokines and Exosomes: Implications for Immunomodulation Studies
}

\author{
Shawna Benjamin-Davalos ${ }^{1}$, Marina Koroleva ${ }^{1}$, Cheryl L. Allen ${ }^{1}$, Marc S. Ernstoff ${ }^{1,2}$ \\ and Shin La Shu ${ }^{1,2 *}$ \\ ${ }^{1}$ Department of Medicine, Roswell Park Comprehensive Cancer Center, Buffalo, NY, United States, 2 ImmunoOncology \\ Branch, Developmental Therapeutics Program, Division of Cancer Treatment and Diagnosis, National Cancer Institute, \\ Frederick, MD, United States
}

\section{OPEN ACCESS}

Edited by: Matthias Kloor,

Heidelberg University Hospital, Germany

Reviewed by:

Daniel Olive,

Aix Marseille Université, France

Lisa Sevenich,

Georg Speyer Haus, Germany

*Correspondence:

Shin La Shu

shushinla@gmail.com;

shinla.shu@nih.gov

Specialty section:

This article was submitted to

Cancer Immunity

and Immunotherapy,

a section of the journal

Frontiers in Immunology

Received: 05 December 2020

Accepted: 19 March 2021

Published: 19 April 2021

Citation:

Benjamin-Davalos S, Koroleva M, Allen CL, Ernstoff MS and Shu SL (2021) Co-Isolation of Cytokines and Exosomes: Implications for Immunomodulation Studies.

Front. Immunol. 12:638111. doi: 10.3389/fimmu.2021.638111
Exosomes play a vital role in intercellular communication and their immunomodulatory potential have become an important focus in cancer research. Various methods have been developed for the isolation although each method differs in the number and purity of exosomes they yield. In melanoma, tumor-derived exosomes drive immunosuppression within the tumor microenvironment. The co-elution of exosomes and soluble factors such as cytokines during isolation, however, make it difficult to ascertain the contribution of exosome cargo, as soluble cytokines are equally capable of immune suppression. In this review we will expound upon the biological relevance that exosome-associated cytokines possess. Furthermore, we discuss the technical challenges that arise during exosome isolation and what this means for further studies into the TME and in vivo work.

Keywords: cytokine co-isolation, exosome-associated cytokines, exosome isolation, tumor microenvironment, melanoma, soluble cytokines, REIUS

\section{INTRODUCTION}

The tumor microenvironment (TME) has been the mainstay of tumor biology research for over 20 years. Cross talk between the immune system and the TME promotes immunosuppression, proangiogenic tendencies, and the inhibition of tumor cell death. Cytokines facilitate intercellular communication between immune cells as well as to other cells within the tumor microenvironment. Therefore, most efforts have focused on the exploitation of the immune system to eliminate cancer using cytokines. Multiple approaches including immunotherapy and cytokine therapy have been used as potential strategies to treat cancer.

Cytokines are categorized as immune-modulating, soluble factors that are less than $30 \mathrm{kDa}$ in size and include chemokines, interferons, interleukins, lymphokines and the tumor necrosis factor family of proteins (1). These molecules function to integrate signals derived from various cell types and to control the growth and activity of their target cell. Cytokines affect almost every biological process, and their downstream effects underlie diseases such as Alzheimer's, autoimmunity, and cancer (2-4).

Cells also release lipid bound vesicles into the extracellular space. These extracellular vesicles (EVs) carry a variety of cargo that includes lipids, proteins and nucleic acids. EVs can be divided into three categories: microvesicles, apoptotic bodies, and exosomes, which differ in the method of 
biogenesis, release, size and content (5). A single protein cannot distinguish between these categories, although their differences in biogenesis contribute to the distinct proteomic profiles between classes. Microvesicles (MVs) are formed from the outward budding of the plasma membrane and range in size from $100 \mathrm{~nm}$ to $1 \mu \mathrm{m}$ in diameter. The exact method of MV formation is not well characterized, but they contain many cytosolic and plasma membrane proteins. MVs are mediators of intercellular communication as their cargo is taken up by recipient cells and can subsequently alter function (6). Apoptotic bodies range in size from $50-5 \mu \mathrm{m}$ and are released by dying cells via blebbing, a hallmark of cell death and the consequence of the disintegration of the cytoskeleton (7). Distinct from other types of EVs, apoptotic bodies contain chromatin, and intact organelles (8).

A subset of EVs ranging in size from 30-150 nm are exosomes which have been implicated in the progression of cancer through the trafficking of bioactive signals, either embedded in their membranes or packaged as payloads. Exosomes are formed from the release of multivesicular bodies into the extracellular space where they are either taken up by cells locally or travel through the bloodstream to more distal sites. Exosomes contain a variety of cytoplasmic proteins as well as nucleic acids (DNA, RNA) and various lipid species capable of translating immunomodulatory effects to cells (9). The secretion of EVassociated cytokines provides an additional mechanism by which cells can maintain specificity and integrity of signaling to distal cells (10). Cytokines possess a high affinity for their corresponding receptors, and thus are effective at concentrations in the picomolar range. Because of this, their secretion is tightly regulated (2). It is now recognized that they may also be packaged into exosomes as exosome-associated cytokines (EACs) (Figure 1) (10). Exosomes are thought to enhance the specificity of cytokine signals through the presence of receptors such as MHCs, tetraspanins and lactadherins in the exosome membrane, which are important for targeting other cell types (11). The release of both free

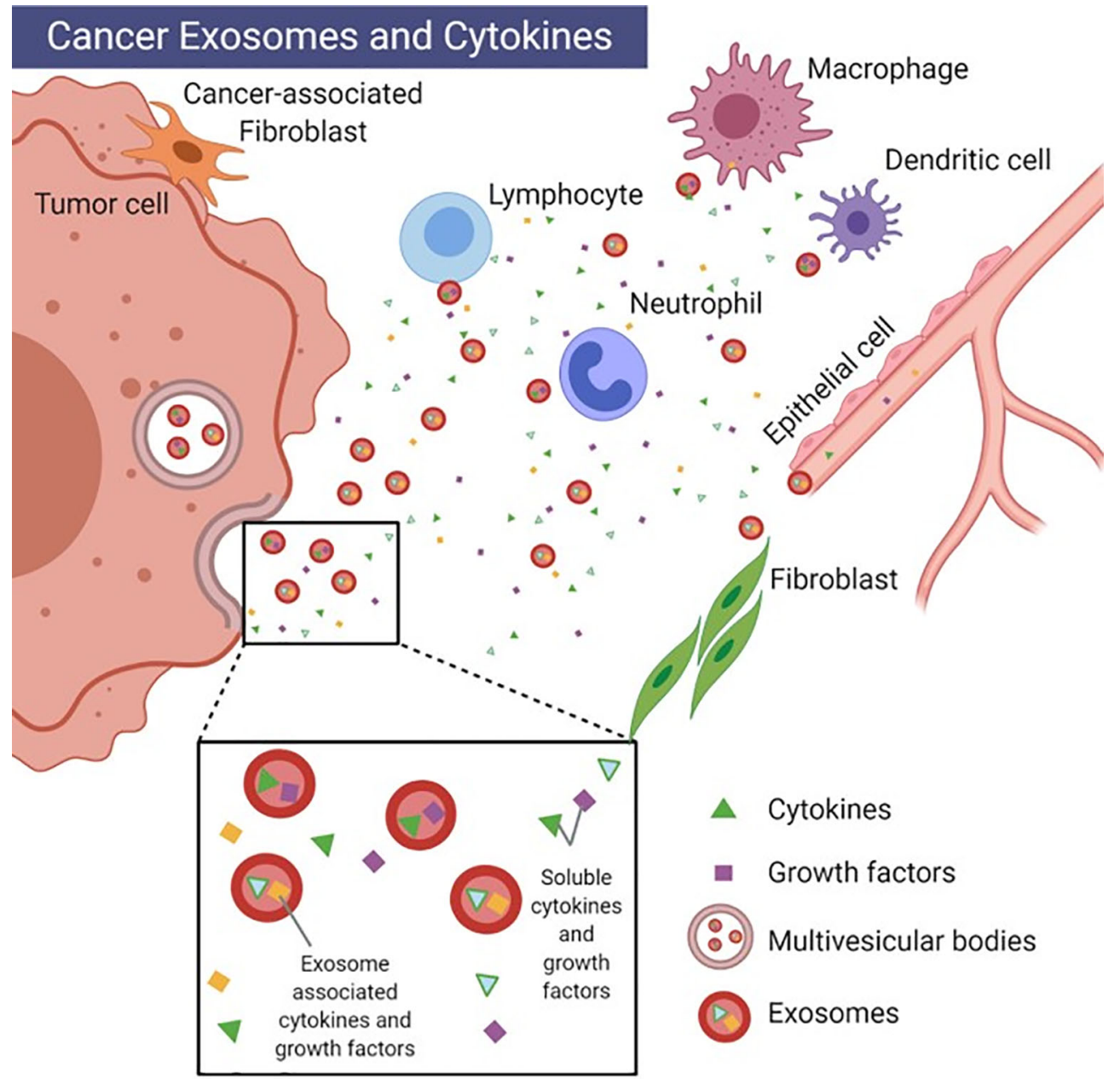

FIGURE 1 | Cancer exosomes and cytokines. Depiction of uni-directional intercellular communication between tumor cell exosomes, immune cells and stromal cells. Image created with BioRender.com. 
and exosome-associated cytokines can potentially complicate in vitro work. Nevertheless, the ability to package a variety of critically bioactive molecules within a stable, lipid membrane-bound vesicle makes exosomes appealing to study as vehicles for therapeutics (12).

With the increasing potential for the use of exosomes clinically, it has become important to optimize exosome isolation methods to ensure maximum purity, yield and reproducibility. Due to the variety of exosome isolation methods utilized, the question arises as to whether current techniques can isolate exosomes devoid of cytokines and other soluble factors, which can confound the causative associations reported in literature. In this review, we will assess the biological role of EACs. Furthermore, we will discuss the technical challenges that preclude us from excluding soluble cytokines from exosome isolates and how this can complicate studies of the TME as well as other in vivo studies.

\section{PRECEDENT FOR MEMBRANE-BOUND CYTOKINES}

Multiple factors including cell origin, health status and environmental factors prior to exosome isolation can impact the variety and quantity of secreted cytokines present in exosome samples (13-15). Estrogen receptor (ER)-negative breast cancer cell lines secrete larger quantities of IL-6 compared to ERpositive cell lines (16). Nutrient deprivation of lung carcinoma (A549) cells for 24 hours showed an increase in the secretion of specific proinflammatory cytokines such as IL-6, IL-8 and chemokine CXCL1 compared to nutrient enriched cells (17). Moreover, subjects exposed to hypoxic conditions within a hypoxic chamber secreted more proinflammatory cytokines than those in standard conditions (18).

To decrease a cytokines' effective range and thereby maintain specificity, many cytokines also function as a membrane-bound protein. Several cytokines and their receptors including IL-1, MCSF, TGF $\beta$, and TNF $\alpha$ have been reported in other studies to exist in both soluble and membrane-bound forms, both which are biologically active (19-23). Most notably, proinflammatory cytokine TNF $\alpha$ was found to also exist in a transmembrane form where it functions as a precursor to soluble TNF $\alpha$ and is able to exert its own cytotoxic activity by acting as a ligand for TNF receptor 2 (TNF-R2) $(21,24)$.

This logic can apply to EVs as well (25). Cytokines may be prepackaged into vesicles as a means of protecting these signals from degradation; however, controversy exists surrounding whether cytokines are displayed on the exosome surface or packaged into the payload. Additionally, the presence of surfacedisplayed cytokines can confound analyses for soluble cytokines. Fitzgerald et al. showed that EACs are either membrane-bound or EV-encapsulated (26). While encapsulated cytokines can be detected by lysing EVs prior to standard detection assays such as ELISA, the detection of membrane-bound cytokines using traditional cytokine detection methods is not as clear. Nine cytokines- IL-6, IL-8, IL-13, IL-16, IP-10, MCP-1, MIP-1 $\alpha$,
MIP-1 $\beta$ and MIP-3 $\alpha$ were found more often in soluble form (26). However, we have recently shown that the exosome isolation method used can impact the presence of soluble cytokines coeluted with exosomes. When corrected for the number of isolated exosomes, the Rapid Exosome Isolation using Ultrafiltration and Size-exclusion chromatography (REIUS) method, led to an 836-fold reduction in 13 cytokines including IL-10 compared to the more commonly used ultracentrifugation (UC) isolation method, suggesting that the cytokines detected may not be physically associated with exosomes (27). The absence of data explicitly showing the association of cytokines to either the EVmembrane or the payload proves to be a major limitation for a number of studies, including our own. Exosomes do demonstrate the capacity to contain membrane-bound cytokines: rheumatoid arthritis synovial fibroblasts (RASF)-derived exosomes induced cytotoxicity in L929 cells (TNF- $\alpha$-sensitized cell line) upon incubation for 24 hours (28). Colloidal gold immunostaining using electron microscopy (EM), was used to confirm the presence of membrane-bound TNF- $\alpha$. Other cytokines with a known membrane-bound form in the originating cell could likely be packaged into the EV membrane. Zhang et al. reported that renal cancer (RC-2) cells transfected with engineered glycolipid-anchorIL-12 packaged this membrane-bound form into exosomes, which reversed tumor exosome-mediated inhibition of T cell activity (29). Again, although inferred, they did not show direct GPI-IL-12 association with exosomes by EM.

Fitzgerald et al. also showed that whether a cytokine will be secreted or packaged into exosomes is dependent upon the biological system and nature of the stimulus (26). Placental villous explants preferentially secreted a subset of cytokines (IL-6, IL-8, IL-15, GRO- $\alpha$, IP-10, MCP-1, MIP-1 $\beta$, MIP-3 $\alpha$, and RANTES) in soluble form, whereas T cells and monocytes in culture produced cytokines in an EV-associated form. Other tissues and body fluids included in the study presented a more even distribution of soluble and EACs. The distribution of cytokines between molecular forms was dramatically changed among activated monocytes stimulated with either lipopolysaccharide (LPS) or Toll-like receptor (TLR3) agonist Poly I:C. Poly I:C-activated monocytes shifted toward the production of more soluble cytokines, demonstrating that the form of expression is not the property of a particular cytokine. How these signals are perceived by target cells and whether or not a difference in the secreted form of cytokines elicits different functions in target cells remains to be addressed. Rana et al. has shown that poly I:C-stimulated keratinocytes could secrete both soluble and EV-associated forms of IL-36 $\gamma$ implying that separate, regulated signaling pathways exist for cytokine secretion (30). The loading of these cytokines into vesicles may involve the function of chaperone proteins as HSP90 was found to be required for the translocation of IL-1 $\beta$ onto vesicle intermediates (31). Differences in biological function have also been demonstrated for soluble versus membrane-bound cytokine receptors. Specifically, soluble IL-6 receptor in complex with IL6 , elicits pro-inflammatory trans-signaling whereas the binding of IL-6 to membrane-bound IL-6R promotes anti-inflammatory downstream effects $(32,33)$. 


\section{QUANTITATIVE LOOK AT CYTOKINE PRESENCE IN EXOSOME ISOLATES}

There is no strong evidence to suggest that exosomes can be isolated with the complete exclusion of cytokines. In 2016, Gardiner et al. showed that $81 \%$ of studies used UC over other isolation methods (34). UC separates EVs using centrifugal force and thus at high speeds, EVs are pelleted while particles that are less dense remain in the supernatant (35). As research on EVs has expanded over the years, additional isolation techniques have been developed. These techniques, which include ultrafiltration (UF), and size exclusion chromatography (SEC), involve separating exosomes based on molecular weight or size/ diameter. In addition, modified methods such as REIUS, affinity-capture based methods, exosome precipitation and microfluidic-based techniques exist as well- each comes with its own advantages and disadvantages as an increase in exosome yield does not necessarily correlate with enhanced exosome purity (Table 1). Immunoaffinity-capture- based techniques are highly specific and result in pure isolations, but is highly dependent on surface marker specificity, which may cause the isolation of a subset of exosomes instead of the full heterogenous composition. As with any antibody-based purification method it is highly sensitive to the concentration of antigen (e.g. exosomes) and this may cause low yield of exosome isolation. Large-scale analyses of heterogeneous exosome populations may become biased toward certain subsets of exosomes if captured on enriched but not exclusive, exosome markers using immunoaffinity capture such as TIM4 and tetraspanins CD63, CD9 and CD81 (39). Phosphatidylserine present on the surface of exosomes has also been proposed as a method for detecting cancer exosomes (45). Exosomal precipitation methods utilize waterexcluding polymers like polyethylene glycol (PEG) in popular commercial products such as ExoQuick (36). These polymers bind water molecules, while less soluble molecules are isolated by centrifugation. This method yields exosomes of low purity and number as contaminants are co-isolated along with EVs (42). Microfluidics-based techniques rapidly isolate exosomes based on their physical and biochemical properties and can address issues of purity through isolating subsets of exosomes similar to affinitybased methods, or by isolating exosomes based on size alone (43, 44). The use of these methods to mitigate cytokine contamination requires further investigation.

Shu et al. compared the soluble cytokine levels present in exosome isolations using REIUS, UC, UF, and SEC methods and found that exosomes isolated from the supernatant of two

TABLE 1 | Summary of existing exosome isolation methods.

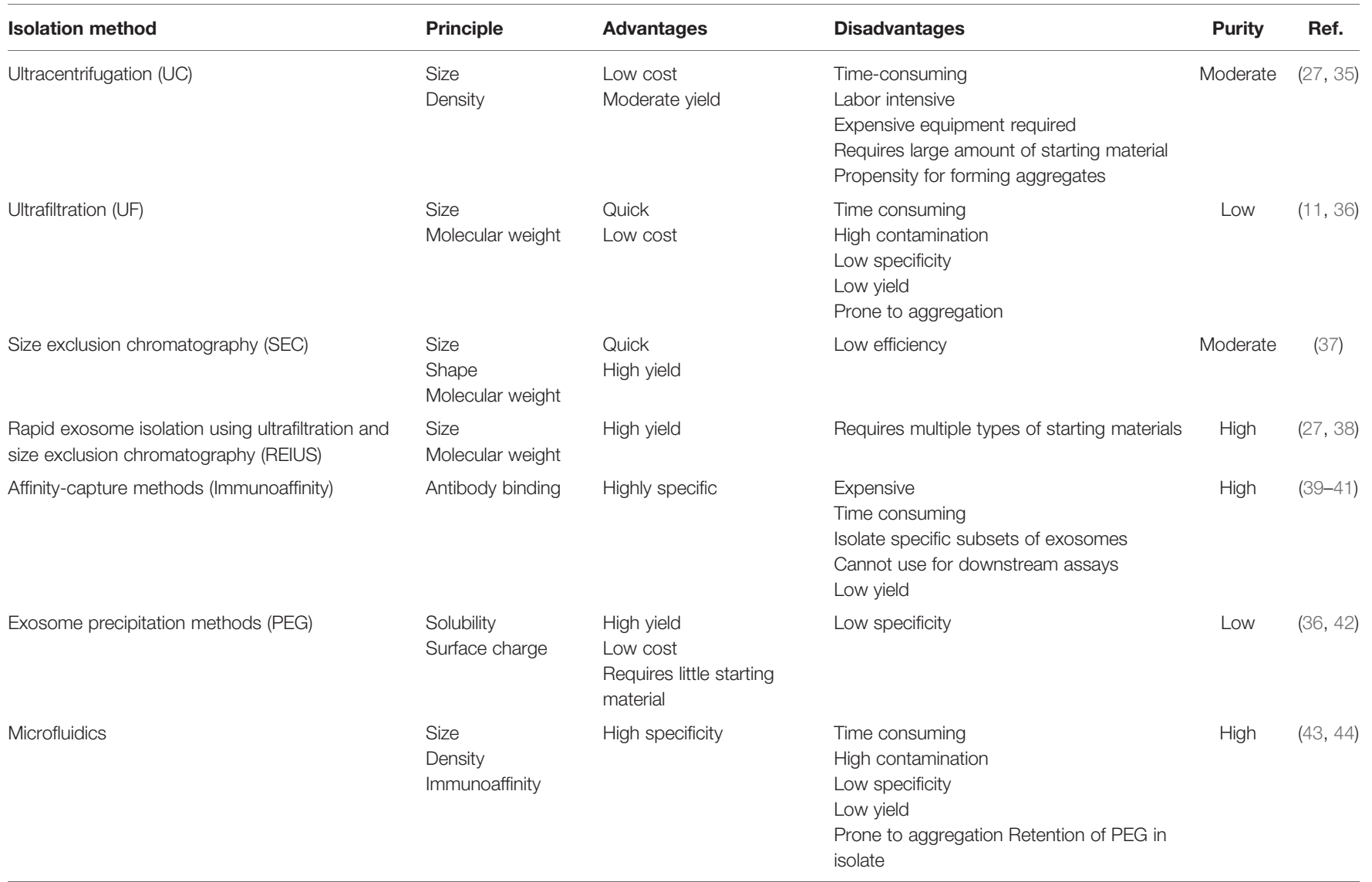

UC, ultracentrifugation; UF, ultrafiltration; SEC, size exclusion chromatography; REIUS, Rapid exosome isolation using ultrafiltration and size exclusion chromatography; PEG, polyethylene glycol. 
melanoma cell lines had varying cytokine levels depending on the isolation method used (26). All methods led to a decrease in the concentration of cytokines detected in exosome isolates compared to the original supernatants, however the REIUS method had the greatest impact in reducing the presence of soluble cytokines. REIUS isolation reduced cytokine concentrations by several logs in comparison to UC and outperformed the use of SEC alone, decreasing IL-10 levels by more than 8 -fold to $97 \mathrm{pg} / \mathrm{ml}$ versus $814 \mathrm{pg} / \mathrm{ml}$. This trend was the same for UF: $96.86 \mathrm{pg} / \mathrm{ml}$ of IL-10 was detected in the REIUS-isolated sample versus $44,450 \mathrm{pg} / \mathrm{ml}$ in the UF flowthrough, demonstrating that the UF step is critical to reducing the concentration of soluble cytokines. IL- 8 and IL-10 were present in high concentrations (cell type-dependent) but overall reduction in concentration was seen for all 13 cytokines tested thus concluding that while REIUS cannot remove all soluble cytokines, they are reduced to a greater extent when compared to other exosome isolation methods, ensuring higher purity without sacrificing exosome yield. The use of additional methods to mitigate soluble cytokine presence requires further investigation.

\section{THE EFFECT OF CYTOKINE CO- ISOLATION ON IN VIVO STUDIES AND THE TME}

The inability to completely exclude cytokines from exosome isolates poses many challenges for downstream applications. Cytokines bear strong affinities for their corresponding receptor. They are also capable of eliciting proliferative and differentiative effects in specific cells among other functions, and thus have been used extensively as a cancer therapeutic. Proinflammatory cytokines such as IFNo, IL-2, IL-10, IL-12, IL15 and GM-CSF have been tested for anti-tumor effects with varying success $(46,47)$. IFN $\alpha$ and IL-2 were the first cytokines to demonstrate antitumor effects in vivo; this observation led to the development of cytokines as anti-tumor monotherapies. Their use in clinical trials, however, has largely been terminated because of their level of toxicity, particularly at higher doses. High dose IL-2 administered to patients with metastatic melanoma had an overall response rate of $16 \%$, but frequently led to the release of other proinflammatory cytokines causing capillary leak syndrome, flu-like symptoms and hypotension (48). In the current time, the expanded use of CAR $T$ cells, which also elicits a clinical cytokine release syndrome, will also impact EV isolation methods. The coelution of cytokines is relevant to those isolating exosomes, particularly when concentrating body fluids using ultrafiltration (UF), which will ultimately increase the concentration of co-isolated cytokines and could lead to toxicity.

Much work has been conducted to elucidate the role of tumor-derived exosomes within the TME. Cytokines such as TNF $\alpha$, TGF $\beta$, CSF-1, CCL2, CCL3, CCL5 and IL- 8 can induce myeloid cell proliferation and promote immunosuppression within the TME. Human melanoma exosomes are also immunosuppressive. Exosomes expressing the checkpoint inhibitor programmed death ligand 1 (PD-L1), are released by melanoma, driving immunosuppression (49). Murine melanoma-derived exosomes were shown to increase proliferation and inhibit cell death of melanoma tumor in mice (50). In addition, HMEX induced cell death and inhibited proliferation in CD8+ $\mathrm{T}$ cells while downregulating NGK2D expression in natural killer (NK) cells (51). We have shown that HMEX exosomes are co-isolated with high concentrations of IL8. The presence of soluble cytokines within exosome isolates cytokines can diminish or amplify the effects of exosomes, leading to erroneous conclusions. Changes in the functionality of exosomes purported to be immunosuppressive for instance, may indicate the presence of soluble cytokines in exosome isolates but improving upon purification methods in order to reduce the presence of soluble cytokines should not interfere with the behavior of the derived exosomes. Moreover, proinflammatory cytokines in exosome isolates can trigger inflammation, which can be damaging or even fatal.

EACs cannot be excluded from eliciting toxic effects. The stability and high biocompatibility of exosomes have made them an attractive vehicle for chemotherapeutics and other drugs. As such, research efforts have focused on determining which cell types are the most ideal source of exosomes. As exosomes embody a similar molecular profile to the originating cell, retention of the lipid and surface protein profile may prove important for proper exosome function $(52,53)$. Exosomes derived from melanoma tumor have been shown to be immunosuppressive through various mechanisms, which include the enhancement of the production of myeloid derived suppressor cells (MDSCs) and the presence of immunosuppressive EACs (40). Conversely, TEX antigen presentation to DCs as well as pro-inflammatory EACs may exacerbate the patient's condition, illustrating the importance of choosing the proper cell-derived exosomes for therapy (54). To combat this issue, plant exosomes mainly derived from fruit and milk are currently being explored as an alternative as they are equally biocompatible and cheaper to produce. Exosomes from grapes did not produce any cytotoxic effects when administered orally to mice and may prove advantageous for administering treatments without unintentional effects (55). The role of using autologous derived human exosomes leveraging their inherent biology has yet to be explored as a therapeutic. However, care must still be taken to ensure that there is minimal presence of other unwanted soluble factors in the exosome product.

\section{DISCUSSION}

Current technical limitations related to the isolation method prevent us from completely removing soluble factors from exosome isolates, making it difficult to distinguish whether downstream effects are due to exosome function or that of the co-isolated cytokines. Moreover, they also prevent us from fully distinguishing between exosomes and other EVs. For instance, microvesicles bud off from the plasma membrane as opposed to being secreted into the extracellular space. As they overlap in size 
with exosomes and share common surface markers and cargo, they are commonly co-eluted with exosomes. These limitations prove detrimental to the understanding of the intricacies of the TME, particularly the contribution of exosomes in the maintenance of the TME.

The TME consists of tumor cells, vascular cells, fibroblasts and immune cells and the subsequent interaction of all these cell types contributes to the progression of cancer. HMEX are immunosuppressive and contain functional EACs $(56,57)$. As tumor cells secrete more exosomes compared to healthy cells, it is possible that the enhanced secretion seen in melanoma cells is a method of providing a large amount of stable, long range signals necessary for establishing a pre-metastatic niche (58). Because exosome production is a conserved process, almost all cell types produce and secrete exosomes.

Exosomes contain specific protein markers corresponding to the originating cell type. Therefore, it would be hard to decipher a mixture of exosomes derived from various cell types from patient blood, which theoretically can serve as a representation of the TME (59). Sharma et al. demonstrated that the separation of melanoma-derived exosomes from non-tumor exosomes in patient plasma was possible using an antibody against a specific epitope of tumor antigen CSPG4, which is expressed in melanoma cells, but not in normal cells. Ultimately, this alludes to the heterogeneity of exosome populations (60). The existence of subpopulations of exosomes that differ in their proteomic and RNA profiles were found to have differential effects on target cells. Ideally, deciphering the TME would be much simpler if signaling between cells was solely unidirectional however, it is more plausible that bi- and multi-directional communication takes place between the tumor and resident cells of the TME as exosomes display a variety of ligands and receptors allowing for their interaction with multiple cell types. This coordinated network of interactions between cells and signals in the form of secreted exosomes, growth factors and cytokines, termed the 'tumor exosome microenvironment' (TExME), underlies tumor progression and deciphering this network may help to predict patient prognosis (Figure 2).

The presence of EACs, specifically those within the payload of exosomes, is strongly supported by the literature. Numerous cytokines that include IL-2, IL-4, IL-10, IL-18, and IL-33, TNF- $\alpha$, TGF- $\beta$, M-CSF were found to be preferentially enriched within exosomes $(26,59,61-63)$. IL-10 in particular has been demonstrated to be exclusively in the exosome payload and is biologically active, inducing mitophagy in kidney tubular epithelial cells (64). Soluble cytokines that have been released extracellularly into supernatant or patient plasma have biological relevance, however, when focusing on the contribution of

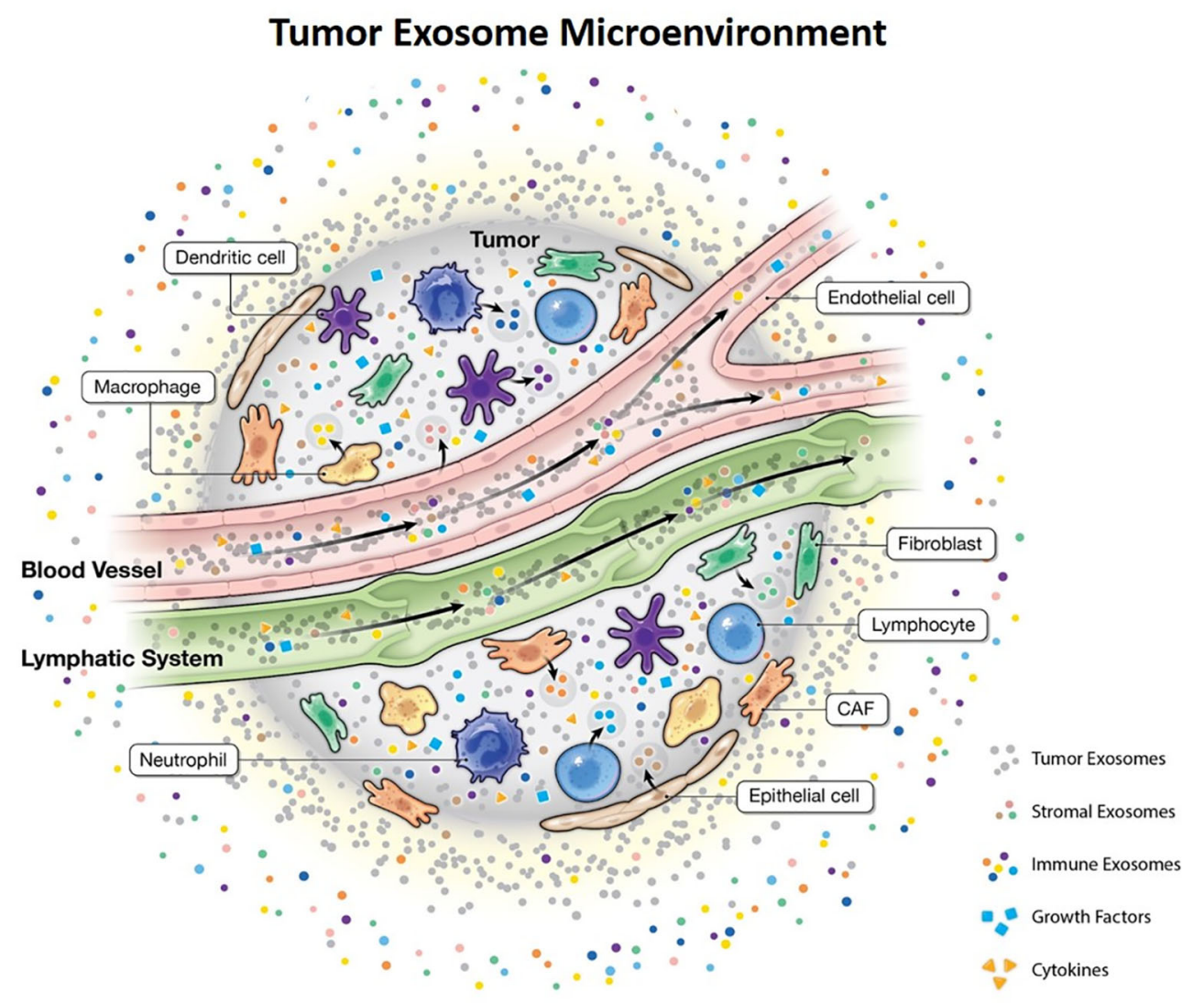

FIGURE 2 | Tumor exosome microenvironment. The orchestrated interaction between various cell types, exosomes and soluble factors. 
exosomes within this network of interactions, the inability to completely remove soluble cytokines from isolates makes it difficult to differentiate them from exosome surface-bound cytokines and only confounds our interpretation of these interactions. At best, combining more than one exosome isolation method may be the way forward for "fit-for-purpose" exosome purification to enhance immunomodulatory studies. For instance, immunoaffinity capture can be used after REIUS to complement the advantages of both methods. The combination of these methods should yield specific populations of exosomes of high purity.

Many factors including cell type and stimulus can affect the level of cytokine presence in an exosome sample as well as the form in which the cytokines are secreted, whether for local or more distant signaling. Understanding these interactions within the milieu that is the 'tumor exosome microenvironment' and further developing technologies to be able to tease them apart is critical to more targeted therapies.

\section{AUTHOR CONTRIBUTIONS}

SB-D: contributed to the general idea, wrote and edited the manuscript, and created figures. MK: contributed ideas to,

\section{REFERENCES}

1. Duitman EH, Orinska Z, Bulfone-Paus S. Mechanisms of cytokine secretion: a portfolio of distinct pathways allows flexibility in cytokine activity. Eur J Cell Biol (2011) 90(6-7):476-83. doi: 10.1016/j.ejcb.2011.01.010

2. Aiello A, Giannessi F, Percario ZA, Affabris E. An emerging interplay between extracellular vesicles and cytokines. Cytokine Growth Factor Rev (2020) 51:49-60. doi: 10.1016/j.cytogfr.2019.12.003

3. Ng A, Tam WW, Zhang MW, Ho CS, Husain SF, McIntyre RS, et al. IL-1 $\beta$, IL-6, TNF- $\alpha$ and CRP in Elderly Patients with Depression or Alzheimer's disease: Systematic Review and Meta-Analysis. Sci Rep (2018) 8(1):12050. doi: 10.1038/s41598-018-30487-6

4. Kunz M, Ibrahim SM. Cytokines and cytokine profiles in human autoimmune diseases and animal models of autoimmunity. Mediators Inflamm (2009) 2009:979258. doi: 10.1155/2009/979258

5. Yáñez-Mó M, Siljander PR, Andreu Z, Zavec AB, Borràs FE, Buzas EI, et al. Biological properties of extracellular vesicles and their physiological functions. J Extracell Vesicles (2015) 4:27066. doi: 10.3402/jev.v4.27066

6. Muralidharan-Chari V, Clancy JW, Sedgwick A, D’Souza-Schorey C. Microvesicles: mediators of extracellular communication during cancer progression. J Cell Sci (2010) 123(Pt 10):1603-11. doi: 10.1242/jcs.064386

7. Battistelli M, Falcieri E. Apoptotic Bodies: Particular Extracellular Vesicles Involved in Intercellular Communication. Biol (Basel) (2020) 9(1):21. doi: 10.3390/biology9010021

8. Jiang L, Paone S, Caruso S, Atkin-Smith GK, Phan TK, Hulett MD, et al. Determining the contents and cell origins of apoptotic bodies by flow cytometry. Sci Rep (2017) 7(1):14444. doi: 10.1038/s41598-017-14305-z

9. Kahlert C, Melo SA, Protopopov A, Tang J, Seth S, Koch M, et al. Identification of double-stranded genomic DNA spanning all chromosomes with mutated KRAS and p53 DNA in the serum exosomes of patients with pancreatic cancer. J Biol Chem (2014) 289(7):3869-75. doi: 10.1074/jbc.C113.532267

10. Barnes BJ, Somerville CC. Modulating Cytokine Production via Select Packaging and Secretion From Extracellular Vesicles. Front Immunol (2020) 11:1040. doi: 10.3389/fimmu.2020.01040

11. Kluszczynska K, Czernek L, Cypryk W, Peczek L, Duchler M. Methods for the Determination of the Purity of Exosomes. Curr Pharm Des (2019) 25 (42):4464-85. doi: 10.2174/1381612825666191206162712 compiled information for manuscript, and edited figures and manuscript. CA: contributed ideas to and edited manuscript. ME: provided expert opinion and input and edited manuscript. SL: conceived the general idea, created figures, and edited the manuscript. All authors contributed to the article and approved the submitted version.

\section{FUNDING}

This work was supported by institutional funds provided by Roswell Park Comprehensive Cancer Center.

\section{ACKNOWLEDGMENTS}

This work was supported by The Katherine Anne Gioia Endowed Chair in Cancer Medicine, Roswell Park Comprehensive Cancer Center. We would like to thank Yana Hammond of ATLAS Studios (https://www.roswellpark.org/ shared-resources/atlas-studios) for helping to create Figure 2's illustration. We also want to thank Dr. Theresa L Whiteside (Department of Pathology, University of Pittsburgh) for her revisions and suggestions regarding this manuscript.

12. Bunggulawa EJ, Wang W, Yin T, Wang N, Durkan C, Wang Y, et al. Recent advancements in the use of exosomes as drug delivery systems. J Nanobiotechnol (2018) 16(1):81. doi: 10.1186/s12951-018-0403-9

13. Kodidela S, Ranjit S, Sinha N, McArthur C, Kumar A, Kumar S. Cytokine profiling of exosomes derived from the plasma of HIV-infected alcohol drinkers and cigarette smokers. PloS One (2018) 13(7):e0201144. doi: 10.1371/journal.pone.0201144

14. Raposo G, Stoorvogel W. Extracellular vesicles: exosomes, microvesicles, and friends. J Cell Biol (2013) 200(4):373-83. doi: 10.1083/ jcb. 201211138

15. Welch JL, Madison MN, Margolick JB, Galvin S, Gupta P, Martinez-Maza O, et al. Effect of prolonged freezing of semen on exosome recovery and biologic activity. Sci Rep (2017) 7:45034. doi: 10.1038/srep45034

16. Sasser AK, Sullivan NJ, Studebaker AW, Hendey LF, Axel AE, Hall BM. Interleukin-6 is a potent growth factor for ER-alpha-positive human breast cancer. FASEB J (2007) 21(13):3763-70. doi: 10.1096/fj.07-8832com

17. Puschel F, Favaro F, Redondo-Pedraza J, Lucendo E, Iurlaro R, Marchetti S, et al. Starvation and antimetabolic therapy promote cytokine release and recruitment of immune cells. Proc Natl Acad Sci USA (2020) 117(18):9932-41. doi: 10.1073/pnas.1913707117

18. Rohm I, Ratka J, Pistulli R, Goebel B, Gecks T, Figulla HR, et al. Impact of Systemic Normobaric Short-Term Hypoxia on Pro-Inflammatory and AntiInflammatory Cytokines in Healthy Volunteers. Clin Lab (2015) 61(8):10539. doi: 10.7754/Clin.Lab.2015.141226

19. Cerretti DP, Wignall J, Anderson D, Tushinski RJ, Gallis BM, Stya M, et al. Human macrophage-colony stimulating factor: alternative RNA and protein processing from a single gene. Mol Immunol (1988) 25(8):761-70. doi: 10.1016/0161-5890(88)90112-5

20. Kurt-Jones EA, Beller DI, Mizel SB, Unanue ER. Identification of a membrane-associated interleukin 1 in macrophages. Proc Natl Acad Sci USA (1985) 82(4):1204-8. doi: 10.1073/pnas.82.4.1204

21. Lopez-Cepero M, Garcia-Sanz JA, Herbert L, Riley R, Handel ME, Podack ER, et al. Soluble and membrane-bound TNF-alpha are involved in the cytotoxic activity of B cells from tumor-bearing mice against tumor targets. J Immunol (1994) 152(7):3333-41.

22. Tran DQ, Andersson J, Wang R, Ramsey H, Unutmaz D, Shevach EM. GARP (LRRC32) is essential for the surface expression of latent TGF-beta on 
platelets and activated FOXP3+ regulatory T cells. Proc Natl Acad Sci USA (2009) 106(32):13445-50. doi: 10.1073/pnas.0901944106

23. Yang CY. Comparative Analyses of the Conformational Dynamics Between the Soluble and Membrane-Bound Cytokine Receptors. Sci Rep (2020) 10 (1):7399. doi: 10.1038/s41598-020-64034-z

24. Georgopoulos S, Plows D, Kollias G. Transmembrane TNF is sufficient to induce localized tissue toxicity and chronic inflammatory arthritis in transgenic mice. J Inflamm (1996) 46(2):86-97.

25. Gao K, Jin J, Huang C, Li J, Luo H, Li L, et al. Exosomes Derived From Septic Mouse Serum Modulate Immune Responses via Exosome-Associated Cytokines. Front Immunol (2019) 10:1560. doi: 10.3389/fimmu.2019.01560

26. Fitzgerald W, Freeman ML, Lederman MM, Vasilieva E, Romero R. Margolis L. A System of Cytokines Encapsulated in ExtraCellular Vesicles. Sci Rep (2018) 8(1):8973. doi: 10.1038/s41598-018-27190-x

27. Shu S, Yang Y, Allen CL, Hurley E, Tung KH, Minderman H, et al. Purity and yield of melanoma exosomes are dependent on isolation method. J Extracell Vesicles (2020) 9(1):1692401. doi: 10.1080/20013078.2019.1692401

28. Zhang HG, Liu C, Su K, Yu S, Zhang L, Zhang S, et al. A membrane form of TNF-alpha presented by exosomes delays $\mathrm{T}$ cell activation-induced cell death. J Immunol (2006) 176(12):7385-93. doi: 10.4049/jimmunol.176.12.7385

29. Zhang Y, Wu XH, Luo CL, Zhang JM, He BC, Chen G. Interleukin-12anchored exosomes increase cytotoxicity of T lymphocytes by reversing the JAK/STAT pathway impaired by tumor-derived exosomes. Int J Mol Med (2010) 25(5):695-700. doi: 10.3892/ijmm_00000393

30. Rana AA, Lucs AV, DeVoti J, Blanc L, Papoin J, Wu R, et al. Poly(I:C) induces controlled release of IL-36gamma from keratinocytes in the absence of cell death. Immunol Res (2015) 63(1-3):228-35. doi: 10.1007/s12026-015-8692-7

31. Zhang M, Kenny SJ, Ge L, Xu K, Schekman R. Translocation of interleukinlbeta into a vesicle intermediate in autophagy-mediated secretion. Elife (2015) 4:e11205. doi: 10.7554/eLife.11205

32. Rose-John S. Interleukin-6 biology is coordinated by membrane bound and soluble receptors. Acta Biochim Pol (2003) 50(3):603-11. doi: 10.18388/ abp.2003_3654

33. Rose-John S. IL-6 trans-signaling via the soluble IL-6 receptor: importance for the pro-inflammatory activities of IL-6. Int J Biol Sci (2012) 8(9):1237-47. doi: $10.7150 /$ ijbs. 4989

34. Gardiner C, Di Vizio D, Sahoo S, Thery C, Witwer KW, Wauben M, et al. Techniques used for the isolation and characterization of extracellular vesicles: results of a worldwide survey. J Extracell Vesicles (2016) 5:32945. doi: 10.3402/ jev.v5.32945

35. Brennan K, Martin K, FitzGerald SP, O'Sullivan J, Wu Y, Blanco A, et al. A comparison of methods for the isolation and separation of extracellular vesicles from protein and lipid particles in human serum. Sci Rep (2020) 10 (1):1039. doi: 10.1038/s41598-020-57497-7

36. Li P, Kaslan M, Lee SH, Yao J, Gao Z. Progress in Exosome Isolation Techniques. Theranostics (2017) 7(3):789-804. doi: 10.7150/thno.18133

37. Mol EA, Goumans MJ, Doevendans PA, Sluijter JPG, Vader P. Higher functionality of extracellular vesicles isolated using size-exclusion chromatography compared to ultracentrifugation. Nanomedicine (2017) 13 (6):2061-5. doi: 10.1016/j.nano.2017.03.011

38. Shu S, Yang Y, Allen CL, Maguire O, Minderman H, Sen A, et al. Metabolic reprogramming of stromal fibroblasts by melanoma exosome microRNA favours a pre-metastatic microenvironment. Sci Rep (2018) 8(1):12905. doi: 10.1038/s41598-018-31323-7

39. Zeringer E, Barta T, Li M, Vlassov AV. Strategies for isolation of exosomes. Cold Spring Harb Protoc (2015) 2015(4):319-23. doi: 10.1101/ pdb.top 074476

40. Frey AB. Myeloid suppressor cells regulate the adaptive immune response to cancer. J Clin Invest (2006) 116(10):2587-90. doi: 10.1172/JCI29906

41. Macias M, Rebmann V, Mateos B, Varo N, Perez-Gracia JL, Alegre E, et al. Comparison of six commercial serum exosome isolation methods suitable for clinical laboratories. Effect in cytokine analysis. Clin Chem Lab Med (2019) 57 (10):1539-45. doi: 10.1515/cclm-2018-1297

42. Alvarez ML, Khosroheidari M, Kanchi Ravi R, DiStefano JK. Comparison of protein, microRNA, and mRNA yields using different methods of urinary exosome isolation for the discovery of kidney disease biomarkers. Kidney Int (2012) 82(9):1024-32. doi: 10.1038/ki.2012.256
43. Dorayappan KDP, Gardner ML, Hisey CL, Zingarelli RA, Smith BQ, Lightfoot MDS, et al. A Microfluidic Chip Enables Isolation of Exosomes and Establishment of Their Protein Profiles and Associated Signaling Pathways in Ovarian Cancer. Cancer Res (2019) 79(13):3503-13. doi: 10.1158/00085472.CAN-18-3538

44. Wang Z, Wu HJ, Fine D, Schmulen J, Hu Y, Godin B, et al. Ciliated micropillars for the microfluidic-based isolation of nanoscale lipid vesicles. Lab Chip (2013) 13(15):2879-82. doi: 10.1039/c3lc41343h

45. Sharma R, Huang X, Brekken RA, Schroit AJ. Detection of phosphatidylserine-positive exosomes for the diagnosis of early-stage malignancies. Br J Cancer (2017) 117(4):545-52. doi: 10.1038/bjc.2017.183

46. Berraondo P, Sanmamed MF, Ochoa MC, Etxeberria I, Aznar MA, PerezGracia JL, et al. Cytokines in clinical cancer immunotherapy. Br J Cancer (2019) 120(1):6-15. doi: 10.1038/s41416-018-0328-y

47. Floros T, Tarhini AA. Anticancer Cytokines: Biology and Clinical Effects of Interferon-alpha2, Interleukin (IL)-2, IL-15, IL-21, and IL-12. Semin Oncol (2015) 42(4):539-48. doi: 10.1053/j.seminoncol.2015.05.015

48. Gallagher DC, Bhatt RS, Parikh SM, Patel P, Seery V, McDermott DF, et al. Angiopoietin 2 is a potential mediator of high-dose interleukin 2-induced vascular leak. Clin Cancer Res (2007) 13(7):2115-20. doi: 10.1158/10780432.CCR-06-2509

49. Poggio M, Hu T, Pai CC, Chu B, Belair CD, Chang A, et al. Suppression of Exosomal PD-L1 Induces Systemic Anti-tumor Immunity and Memory. Cell (2019) 177(2):414-27.e13. doi: 10.1016/j.cell.2019.02.016

50. Matsumoto A, Takahashi Y, Nishikawa M, Sano K, Morishita M, Charoenviriyakul C, et al. Accelerated growth of B16BL6 tumor in mice through efficient uptake of their own exosomes by B16BL6 cells. Cancer Sci (2017) 108(9):1803-10. doi: 10.1111/cas.13310

51. Sharma P, Diergaarde B, Ferrone S, Kirkwood JM, Whiteside TL. Melanoma cell-derived exosomes in plasma of melanoma patients suppress functions of immune effector cells. Sci Rep (2020) 10(1):92. doi: 10.1038/s41598-01956542-4

52. Frydrychowicz M, Kolecka-Bednarczyk A, Madejczyk M, Yasar S, Dworacki G. Exosomes - structure, biogenesis and biological role in nonsmall-cell lung cancer. Scand J Immunol (2015) 81(1):2-10. doi: 10.1111/ sji. 12247

53. Luan X, Sansanaphongpricha K, Myers I, Chen H, Yuan H, Sun D. Engineering exosomes as refined biological nanoplatforms for drug delivery. Acta Pharmacol Sin (2017) 38(6):754-63. doi: 10.1038/aps.2017.12

54. Wolfers J, Lozier A, Raposo G, Regnault A, Thery C, Masurier C, et al. Tumor-derived exosomes are a source of shared tumor rejection antigens for CTL cross-priming. Nat Med (2001) 7(3):297-303. doi: $10.1038 / 85438$

55. Ju S, Mu J, Dokland T, Zhuang X, Wang Q, Jiang H, et al. Grape exosome-like nanoparticles induce intestinal stem cells and protect mice from DSS-induced colitis. Mol Ther (2013) 21(7):1345-57. doi: 10.1038/mt.2013.64

56. Chen G, Huang AC, Zhang W, Zhang G, Wu M, Xu W, et al. Exosomal PD-L1 contributes to immunosuppression and is associated with anti-PD-1 response. Nature (2018) 560(7718):382-6. doi: 10.1038/s41586-018-0392-8

57. Whiteside TL. Immune modulation of T-cell and NK (natural killer) cell activities by TEXs (tumour-derived exosomes). Biochem Soc Trans (2013) 41 (1):245-51. doi: 10.1042/BST20120265

58. Parolini I, Federici C, Raggi C, Lugini L, Palleschi S, De Milito A, et al. Microenvironmental $\mathrm{pH}$ is a key factor for exosome traffic in tumor cells. J Biol Chem (2009) 284(49):34211-22. doi: 10.1074/jbc.M109.041152

59. Fitzgerald W, Gomez-Lopez N, Erez O, Romero R, Margolis L. Extracellular vesicles generated by placental tissues ex vivo: A transport system for immune mediators and growth factors. Am J Reprod Immunol (2018) 80(1):e12860. doi: $10.1111 /$ aji.12860

60. Sharma P, Ludwig S, Muller L, Hong CS, Kirkwood JM, Ferrone S, et al. Immunoaffinity-based isolation of melanoma cell-derived exosomes from plasma of patients with melanoma. J Extracell Vesicles (2018) 7(1):1435138. doi: $10.1080 / 20013078.2018 .1435138$

61. Ringuette Goulet C, Bernard G, Tremblay S, Chabaud S, Bolduc S, Pouliot F. Exosomes Induce Fibroblast Differentiation into Cancer-Associated Fibroblasts through TGFbeta Signaling. Mol Cancer Res (2018) 16(7):1196204. doi: 10.1158/1541-7786.MCR-17-0784 
62. Yaddanapudi K, Meng S, Whitt AG, Al Rayyan N, Richie J, Tu A, et al. Exosomes from GM-CSF expressing embryonic stem cells are an effective prophylactic vaccine for cancer prevention. Oncoimmunology (2019) 8 (3):1561119. doi: 10.1080/2162402X.2018.1561119

63. Jung HH, Kim JY, Lim JE, Im YH. Cytokine profiling in serum-derived exosomes isolated by different methods. Sci Rep (2020) 10(1):14069. doi: 10.1038/s41598-020-70584-z

64. Tang TT, Wang B, Wu M, Li ZL, Feng Y, Cao JY, et al. Extracellular vesicleencapsulated IL-10 as novel nanotherapeutics against ischemic AKI. Sci Adv (2020) 6(33):eaaz0748. doi: 10.1126/sciadv.aaz0748
Conflict of Interest: The authors declare that the research was conducted in the absence of any commercial or financial relationships that could be construed as a potential conflict of interest.

Copyright $\odot 2021$ Benjamin-Davalos, Koroleva, Allen, Ernstoff and Shu. This is an open-access article distributed under the terms of the Creative Commons Attribution License (CC BY). The use, distribution or reproduction in other forums is permitted, provided the original author(s) and the copyright owner(s) are credited and that the original publication in this journal is cited, in accordance with accepted academic practice. No use, distribution or reproduction is permitted which does not comply with these terms. 